\title{
Almost automorphic solutions for shunting inhibitory cellular neural networks with time-varying delays
}

\author{
Changjin $\mathrm{Xu}^{{ }^{*}}$ and Maoxin Liao ${ }^{2}$
}

*Correspondence: xcj403@126.com

${ }^{1}$ Guizhou Key Laboratory

of Economics System

Simulation, Guizhou

University of Finance

and Economics,

Guiyang 550004, People's

Republic of China

Full list of author information

is available at the end of the article

\begin{abstract}
This paper is concerned with the shunting inhibitory cellular neural networks with time-varying delays. Under some suitable conditions, we establish some criteria on the existence and global exponential stability of the almost automorphic solutions of the networks. Numerical simulations are given to support the theoretical findings.

Keywords: Shunting inhibitory cellular neural networks, Almost automorphic solution, Exponential stability, Time-varying delay

Mathematics Subject Classification: 39A60, 39A12
\end{abstract}

\section{Background}

It is well known that shunting inhibitory cellular neural networks with delay have been successfully applied in variety of areas such as signal processing, pattern recognition, chemical processes, nuclear reactors, biological systems, static image processing, associative memories, optimization problems and so on (Roska and Chua 1992; Chua and Yang 1988a, b; Chua and Roska 1990; Zhang and Shao 2013). In the past decades, there have been extensive results on the dynamical behavior of shunting inhibitory cellular neural networks networks such as the existence and stability of equilibrium points, periodic solutions, almost periodic solutions and anti-periodic solutions, etc. We refer the reader to (Wang et al. 2014a, b; Song et al. 2012; Fan and Shao 2010; Li and Wang 2012; Xia et al. 2007; Peng and Wang 2013; Bouzerdoum and Pinter 1993; Chen and Zhao 2008; Xia et al. 2007; Shao 2008; Yang and Cao 2007; Zhang 2013; Huang et al. 2010).

In particular, we shall point out that almost periodicity is universal than periodicity in real word, moreover, almost automorphic functions, which were introduced by Bochner, are much more general than almost periodic functions. The almost automorphic solutions have potential applications in various fields such as linear and nonlinear evolution equations, integro-differential and functional-differential equations, dynamical systems and so on (Cuevas et al. 2012; N'Gérékata 2005). Almost automorphic solutions in the context of differential equations were studied by several authors. We refer the reader to (Hilger 1990; N'Guérékata 2004, 2005; Goldstein and N'Guérékata 2005; Ezzinbi et al. 2007; Chérif and Nahia 2013; Chérif 2014; Wang and Li 2013; Lizama and Mesquita

(C) $2015 \mathrm{Xu}$ and Liao. This article is distributed under the terms of the Creative Commons Attribution 4.0 International License (http://creativecommons.org/licenses/by/4.0/), which permits unrestricted use, distribution, and reproduction in any medium, provided you give appropriate credit to the original author(s) and the source, provide a link to the Creative Commons license, and indicate if changes were made. 
2013). However, to the best of our knowledge, there are very few papers published on the almost automorphic solutions of shunting inhibitory cellular neural networks with timevarying delays (Li and Yang 2014; Abbas et al. 2014).

Inspired by the discuss above, in this paper, we consider the following shunting inhibitory cellular neural networks with time-varying delays

$$
\begin{aligned}
x_{i j}^{\prime}(t)= & -a_{i j}(t) x_{i j}(t)+\sum_{C_{k l} \in N_{r}(i, j)} C_{i j}^{k l}(t) f\left(x_{k l}\left(t-\tau_{k l}(t)\right)\right) x_{i j}(t) \\
& +\sum_{C_{k l} \in N_{q}(i, j)} B_{i j}^{k l}(t) \int_{0}^{\infty} K_{i j}(u) g\left(x_{k l}(t-u)\right) d u x_{i j}(t)+L_{i j}(t),
\end{aligned}
$$

where $C_{i j}$ denotes the cell at the $(i, j)$ position of the lattice. The $r$-neighborhood $N_{r}(i, j)$ of $C_{i j}$ is given as

$$
N_{r}(i, j)=\left\{C_{k l}: \max (|k-i|,|l-j|) \leq r, \quad 1 \leq k \leq m, 1 \leq l \leq n\right\},
$$

where $i=1,2, \ldots, m, j=1,2, \ldots, n, N_{q}(i, j)$ is similarly specified, $x_{i j}$ is the activity of the cell $C_{i j}, L_{i j}(t)$ is the external input to $C_{i j}$, the function $a_{i j}(t)>0$ represents the passive decay rate of the cell activity, $C_{i j}^{k l}$ and $B_{i j}^{k l}$ are the connection or coupling strength of postsynaptic activity of the cell transmitted to the cell $C_{i j}$, and the activity functions $f($.) and $g($.$) are continuous functions representing the output or firing rate of the cell C_{k l}$, and $\tau_{k l}(t) \geq 0$ corresponds to the transmission delay, the kernel $K_{i j}$ is a piecewise continuous integrable function and satisfies

$$
\int_{-\infty}^{t} K_{i j}(t-s) d s=1, \int_{0}^{\infty} K_{i j}(s) e^{\alpha s} d s<+\infty, \quad \alpha>0 .
$$

It is easy to see that system (1) is equivalent to the form

$$
\begin{aligned}
x_{i j}^{\prime}(t)= & -a_{i j}(t) x_{i j}(t)+\sum_{C_{k l} \in N_{r}(i, j)} C_{i j}^{k l}(t) f\left(x_{k l}\left(t-\tau_{k l}(t)\right)\right) x_{i j}(t) \\
& +\sum_{C_{k l} \in N_{q}(i, j)} B_{i j}^{k l}(t) \int_{-\infty}^{t} K_{i j}(t-u) g\left(x_{k l}(u)\right) d u x_{i j}(t)+L_{i j}(t) .
\end{aligned}
$$

The main aim of this paper is to establish a set of sufficient conditions for the existence and exponential stability of almost automorphic solutions for model (3).

The remainder of the paper is organized as follows. In "Preliminary results", we introduce the basic properties of almost automorphic functions, some necessary notations, definitions and preliminaries which will be used later. In "Existence of almost automorphic solutions", we present some sufficient conditions for the existence of almost automorphic solutions of (3). Some sufficient conditions on the global exponential stability of almost automorphic solutions of (3) are established in "Exponential stability of almost automorphic solutions". An example is given to illustrate the effectiveness of the obtained results in "Numerical example" . A brief conclusion is drawn in "Conclusions". 


\section{Preliminary results}

In this section, we would like to recall some basic definitions and lemmas related to the concept of almost automorphy which shall come into play later on.

Definition 2.1 (Bochner 1962) A continuous function $f: \mathbb{R} \rightarrow \mathbb{R}^{n}$ is said to be almost automorphic if for every sequence of real numbers $\left(s_{n}^{\prime}\right)_{n \in \mathbb{N}}$, there exists a subsequence $\left(s_{n}\right)_{n \in \mathbb{N}}$ such that $g(t):=\lim _{n \rightarrow \infty} f\left(t+s_{n}\right)$ is well defined for each $t \in \mathbb{R}$, and $\lim _{n \rightarrow \infty} g\left(t-s_{n}\right)=f(t)$ for each $t \in \mathbb{R}$.

Remark 2.1 (Chérif 2014) Note that the function $g$ in definition above is measurable but not necessarily continuous. Moreover, if $g$ is continuous, then $\mathrm{f}$ is uniformly continuous. Besides, if the convergence above is uniform in $t \in \mathbb{R}$, then $f$ is almost periodic. Denote by $A A\left(\mathbb{R}, \mathbb{R}^{n}\right)$ the collection of all almost automorphic functions, then

$$
A P\left(\mathbb{R}, \mathbb{R}^{n}\right) \subset A A\left(\mathbb{R}, \mathbb{R}^{n}\right) \subset B C\left(\mathbb{R}, \mathbb{R}^{n}\right),
$$

where $A P\left(\mathbb{R}, \mathbb{R}^{n}\right)$ and $B C\left(\mathbb{R}, \mathbb{R}^{n}\right)$ are respectively the collection of all almost periodic functions and the set of bounded continuous functions from $\mathbb{R}$ to $\mathbb{R}^{n}$.

Lemma 2.1 (N'Guérékata 2005) For all $f, f_{1}, f_{2} \in A A\left(\mathbb{R}, \mathbb{R}^{n}\right)$, one has

1. $f_{1}+f_{2} \in A A\left(\mathbb{R}, \mathbb{R}^{n}\right)$.

2. $\lambda f \in A A\left(\mathbb{R}, \mathbb{R}^{n}\right)$ for any scalar $\lambda \in \mathbb{R}$.

3. $f_{\alpha} \in A A\left(\mathbb{R}, \mathbb{R}^{n}\right)$, where $f_{\alpha}: \mathbb{R} \rightarrow X$ is defined by $f_{\alpha}()=.f(.+\alpha)$.

4. Let $f \in A A\left(\mathbb{R}, \mathbb{R}^{n}\right)$, then the range $R_{f}:=\{f(t), t \in \mathbb{R}\}$ is relatively compact in $X$, thus $f$ is bounded in norm.

5. If $f_{n} \rightarrow f$ uniformly on $\mathbb{R}$, where $f_{n} \in A A\left(\mathbb{R}, \mathbb{R}^{n}\right)$, then $f \in A A\left(\mathbb{R}, \mathbb{R}^{n}\right)$.

6. $\left(A A\left(\mathbb{R}, \mathbb{R}^{n}\right),\|.\|_{\infty}\right)$ is a Banach space.

Definition 2.2 A function $f \in C\left(\mathbb{R} \times \mathbb{R}^{n}, \mathbb{R}^{n}\right)$ is said to be almost automorphic in $t \in \mathbb{R}$ for each $x \in X$ if for every sequence of real numbers $\left(s_{n}^{\prime}\right)_{n \in \mathbb{N}}$, there exists a subsequence $\left(s_{n}\right)_{n \in \mathbb{N}}$ such that $g(t, x):=\lim _{n \rightarrow \infty} f\left(t+s_{n}, x\right)$ is well defined for each $t \in \mathbb{R}$, $x \in \mathbb{R}^{n}$ and $\lim _{n \rightarrow \infty} g\left(t-s_{n}, x\right)=f(t, x)$ for each $t \in \mathbb{R}, x \in \mathbb{R}^{n}$. The collection of such functions will be denoted by $A A\left(\mathbb{R} \times \mathbb{R}^{n}, \mathbb{R}^{n}\right)$.

Lemma 2.2 (Diagana et al. 2008) Let $f: \mathbb{R} \times \mathbb{R}^{n} \rightarrow \mathbb{R}^{n}$ be an almost automorphic function in $t \in \mathbb{R}$ for each $x \in \mathbb{R}^{n}$ and assume that $f$ satisfies a Lipschitz condition in $x$ uniformly in $t \in \mathbb{R}$. Let $\varphi: \mathbb{R} \rightarrow \mathbb{R}^{n}$ be an almost automorphic function. Then the function $\phi: t \mapsto \phi(t)=f(t, \varphi(t))$ is almost automorphic.

Definition 2.3 The almost automorphic solution $x_{i j}()=.\left(x_{11}(),. x_{12}(),. \ldots, x_{m n}().\right)$ of SICNNs is said to be globally exponentially stable, if, for any solution $x()=.\left(x_{11}(),. x_{12}(),. \cdots, x_{m n}().\right)$, there exist constants $M>0$ and $\mu>0$ such that for all $t \in \mathbb{R}$,

$$
\left\|x^{*}(t)-x(t)\right\| \leq M e^{-\mu t}
$$


Lemma 2.3 (Hale 1977) (The upper-right Dini derivative) Let $f: \mathbb{R} \rightarrow \mathbb{R}$ be a continuous function, then the upper-right Dini derivative $\frac{D^{+} f(t)}{d t}$ is defined by

$$
\frac{D^{+} f(t)}{d t}=\varlimsup_{h \rightarrow 0^{+}} \frac{f(t+h)-f(t)}{h} .
$$

Remark 2.2 (Abbas et al. 2014) The upper-right Dini derivative $\frac{D^{+} f(t)}{d t}$ of $|f(t)|$ is given by

$$
\frac{D^{+} V|f(t)|}{d t}=\operatorname{sign}(f(t)) \frac{d f(t)}{d t},
$$

where $\operatorname{sign}($.$) is the signum function.$

\section{Existence of almost automorphic solutions}

In this section, we will establish sufficient conditions on the existence of almost automorphic solutions of (1). Denote

$$
\Lambda=\{11,12,1 n, 21,22, \ldots, 2 n, m n\}, \quad \tau=\max _{1 \leq k \leq m, 1 \leq l \leq n}\left\{\tau_{k l}(t)\right\}
$$

Throughout this paper, we make the assumptions as follows.

(H1) There exists constants $L_{f}>0, L_{g}>0, M_{f}>0$ and $M_{g}>0$ such that for all $u, v \in \mathbb{R}$,

$|f(u)-f(v)| \leq L_{f}|u-v|,|g(u)-g(v)| \leq L_{g}|u-v|,|f(u)| \leq M_{f}, \quad|g(u)| \leq M_{g}$.

Furthermore, $f(0)=g(0)=0$.

(H2) For $i j \in \Lambda, L()=.\left(L_{11}(),. L_{12}(),. \cdots, L_{m n}().\right) \in A A\left(\mathbb{R}, \mathbb{R}^{m+n}\right)$ and $a_{i j}(t), C_{i j}^{k l}$ and $B_{i j}^{k l}$ all almost automorphic.

(H3) For $i j \in \Lambda$,

$\gamma=\max _{i j \in \Lambda} \sup _{t \in \mathbb{R}}\left\{\frac{\sum_{C_{k l} \in N_{r}(i, j)}\left|C_{i j}^{k l}(t)\right| L_{f}+\frac{M}{u} \sum_{C_{k l} \in N_{q}(i, j)}\left|B_{i j}^{k l}(t)\right| L_{g}}{a^{-}}\right\}<1, \quad \frac{\|L\|_{\infty}}{a^{-}(1-\gamma)}<1$,

where $a_{i j}^{-}=\min _{t \in \mathbb{R}} a_{i j}(t), a^{-}=\min _{i j \in \Lambda} a_{i j}^{-}$.

(H4) For $i j \in \Lambda, \max _{i j \in \Lambda} \sup _{s \in \mathbb{R}}\left\{\frac{\Pi_{i j}}{a^{-}}\right\}<1$, where

$\Pi_{i j}=\sum_{C_{k l} \in N_{r}(i, j)}\left|C_{i j}^{k l}(s)\right|\left(M_{f}+L_{f}\right)+\sum_{C_{k l} \in N_{q}(i, j)}\left|B_{i j}^{k l}(s)\right|\left(1+\frac{\|L\|_{\infty}}{a^{-}(1-\gamma)}\right) L_{g} \int_{0}^{\infty}\left|K_{i j}(u)\right| d u$.

(H5) The kernel $K_{i j}($.$) is almost automorphic and there exist M>0$ and $u>0$ such that

$\left|K_{i j}(t)\right| \leq M e^{-u t}$

Lemma 3.1 Suppose that assumptions (H1) and (H5) hold and $x_{i j}(.) \in A A(\mathbb{R}, \mathbb{R})$, then

$$
\phi: t \mapsto \int_{-\infty}^{t} K_{i j}(t-s) g\left(x_{k l}(s)\right) d s
$$

belongs to $A A(\mathbb{R}, \mathbb{R})$. 
Proof By the composition theorem of almost automorphic functions (N'Guérékata 2005), the functions $\psi: s \mapsto g\left(x_{k l}(s)\right)$ belongs to $A A(\mathbb{R}, \mathbb{R})$ whenever $x_{k l} \in A A\left(\mathbb{R}, \mathbb{R}^{m+n}\right)$. Now, let $\left(s_{n}^{\prime}\right)$ be a sequence of real numbers. By $(\mathrm{H} 5)$, we can extract a subsequence $\left(s_{n}\right)$ of $\left(s_{n}^{\prime}\right)$ such that for all $t, s \in \mathbb{R}$,

$$
\lim _{n \rightarrow+\infty} K_{i j}\left(t-s+s_{n}\right)=K_{i j}^{1}(t-s), \quad \lim _{n \rightarrow+\infty} K_{i j}^{1}\left(t-s-s_{n}\right)=K_{i j}(t-s),
$$

and

$$
\lim _{n \rightarrow+\infty} \psi\left(t+s_{n}\right)=\psi^{1}(t), \quad \lim _{n \rightarrow+\infty} \psi^{1}\left(t-s_{n}\right)=\psi(t)
$$

Define

$$
\phi^{1}: t \mapsto \int_{-\partial}^{t} K_{i j}(t-s) \psi^{1}(s) d s .
$$

obviously,

$$
\begin{aligned}
\phi^{1}\left(t+s_{n}\right)-\phi^{1}(t) & =\int_{-\infty}^{t+s_{n}} K_{i j}\left(t-s+s_{n}\right) \psi(s) d s-\int_{-\infty}^{t} K_{i j}(t-s) \psi^{1}(s) d s \\
& =\int_{-\infty}^{t} K_{i j}(t-u) \psi\left(u+s_{n}\right) d u-\int_{-\infty}^{t} K_{i j}(t-s) \psi^{1}(s) d s \\
& =\int_{-\infty}^{t} K_{i j}(t-u)\left|\psi\left(u+s_{n}\right)-\psi^{1}(s)\right| d s \\
& =\int_{-\infty}^{t} M e^{-(t-s) u}\left|\psi\left(u+s_{n}\right)-\psi^{1}(s)\right| d s .
\end{aligned}
$$

In view of Lebesgue Dominated Convergence Theorem and (H2), we have for all $t \in \mathbb{R}$,

$$
\lim _{n \rightarrow \infty} \phi\left(t+s_{n}\right)=\phi^{1}(t) .
$$

Similarly we have for all $t \in \mathbb{R}$,

$$
\lim _{n \rightarrow \infty} \phi\left(t-s_{n}\right)=\phi(t),
$$

which implies that

$$
\phi: t \mapsto \int_{-\infty}^{t} K_{i j}(t-s) g\left(x_{k l}(s)\right) d s
$$

belongs to $A A(\mathbb{R}, \mathbb{R})$. The proof of Lemma 3.1 is completed.

Define the nonlinear operator $\Phi$ by: for each $\varphi A A \in\left(\mathbb{R}, \mathbb{R}^{m+n}\right)$,

$$
\begin{aligned}
(\Phi \varphi)(t)= & \operatorname{col}\left\{\int _ { - \infty } ^ { t } e ^ { - \int _ { s } ^ { t } a _ { i j } ( u ) d u } \left[\sum_{C_{k l} \in N_{r}(i, j)} C_{i j}^{k l}(s) f\left(\varphi_{k l}\left(s-\tau_{k l}(s)\right)\right) \varphi_{i j}(s)\right.\right. \\
& \left.\left.+\sum_{C_{k l} \in N_{q}(i, j)} B_{i j}^{k l}(s) \int_{0}^{\infty} K_{i j}(u) g\left(\varphi_{k l}(s-u)\right) d u \varphi_{i j}(s)+L_{i j}(s)\right] d s\right\} .
\end{aligned}
$$


Lemma 3.2 If $(H 1-H 3)$ are satisfied. Then $\Phi$ maps $A A\left(\mathbb{R}, \mathbb{R}^{m+n}\right)$ into itself.

Proof First of all, let us check that $\Phi$ is well defined. By Lemma 2.1, we know that the space $A A\left(\mathbb{R}, \mathbb{R}^{m+n}\right)$ is translation invariant. Besides, by Lemmas 2.2 and Lemma 3.1, we can conclude that the function

$$
\begin{aligned}
\Psi_{i j}: s \mapsto & \sum_{C_{k l} \in N_{r}(i, j)} C_{i j}^{k l}(s) f\left(\varphi_{k l}\left(s-\tau_{k l}(s)\right)\right) \varphi_{i j}(s) \\
& +\sum_{C_{k l} \in N_{q}(i, j)} B_{i j}^{k l}(s) \int_{0}^{\infty} K_{i j}(u) g\left(\varphi_{k l}(s-u)\right) d u \varphi_{i j}(s)+L_{i j}(s)
\end{aligned}
$$

belongs to $A A(\mathbb{R}, \mathbb{R})$. Then (4) can be rewritten as

$$
(\Phi \varphi)(t)=\operatorname{col}\left\{\int_{-\infty}^{t} e^{-\int_{s}^{t} a_{i j}(u) d u} \Psi_{i j} d s\right\} .
$$

Let $\left(s_{n}^{\prime}\right)$ be a sequence of real numbers. By $(\mathrm{H} 4)$ we can extract a subsequence $\left(s_{n}\right)$ of $\left(s_{n}^{\prime}\right)$ such that for all $t, s \in \mathbb{R}$,

$$
\lim _{n \rightarrow+\infty} a_{i j}\left(t+s_{n}\right)=a_{i j}^{1}(t), \lim _{n \rightarrow+\infty} a_{i j}^{1}\left(t-s_{n}\right)=a_{i j}(t)
$$

and

$$
\lim _{n \rightarrow+\infty} \Psi_{i j}\left(t+s_{n}\right)=\Psi_{i j}^{1}(t), \lim _{n \rightarrow+\infty} \Psi_{i j}^{1}\left(t-s_{n}\right)=\Psi_{i j}(t)
$$

Define

$$
\left(\Phi^{1} \varphi\right)(t):=\int_{-\infty}^{t} e^{-\int_{s}^{t} a_{i j}^{1}(u) d u} \Psi_{i j}(s) d s .
$$

Then

$$
\begin{aligned}
&\left(\Phi^{1} \varphi\right)\left(t+s_{n}\right)-\left(\Phi^{1} \varphi\right)(t) \\
&=\int_{-\infty}^{t+s_{n}} e^{-\int_{s}^{t+s_{n}} a_{i j}(u) d u} \Psi_{i j}(s) d s-\int_{-\infty}^{t} e^{-\int_{s}^{t} a_{i j}^{1}(u) d u} \Psi_{i j}^{1}(s) d s \\
&=\int_{-\infty}^{t+s_{n}} e^{-\int_{s-s_{n}}^{t} a_{i j}\left(u+s_{n}\right) d u} \Psi_{i j}(s) d s-\int_{-\infty}^{t} e^{-\int_{s}^{t} a_{i j}^{1}(u) d u} \Psi_{i j}^{1}(s) d s \\
&=\int_{-\infty}^{t} e^{-\int_{\theta}^{t} a_{i j}\left(u+s_{n}\right) d u} \Psi_{i j}\left(\theta+s_{n}\right) d \theta-\int_{-\infty}^{t} e^{-\int_{s}^{t} a_{i j}^{1}(u) d u} \Psi_{i j}^{1}(s) d s \\
&=\int_{-\infty}^{t} e^{-\int_{\theta}^{t} a_{i j}\left(u+s_{n}\right) d u} \Psi_{i j}\left(\theta+s_{n}\right) d \theta-\int_{-\infty}^{t} e^{-\int_{\theta}^{t} a_{i j}^{1}\left(u+s_{n}\right) d u} \Psi_{i j}^{1}(\theta) d \theta \\
&+\int_{-\infty}^{t} e^{-\int_{\theta}^{t} a_{i j}\left(u+s_{n}\right) d u} \Psi_{i j}^{1}(\theta) d \theta-\int_{-\infty}^{t} e^{-\int_{\theta}^{t} a_{i j}^{1}(u) d u} \Psi_{i j}^{1}(\theta) d \theta \\
&= \int_{-\infty}^{t} e^{-\int_{\theta}^{t} a_{i j}\left(u+s_{n}\right) d u}\left(\Psi_{i j}\left(s+s_{n}\right)-\Psi_{i j}^{1}(s)\right) d s \\
&-\int_{-\infty}^{t}\left(e^{-\int_{\theta}^{t} a_{i j}\left(u+s_{n}\right) d u}-e^{-\int_{s}^{t} a_{i j}^{1}(u) d u}\right) \Psi_{i j}^{1}(s) d s .
\end{aligned}
$$


Applying the Lebesgue DominatedConvergence Theorem, we have

$$
\lim _{n \rightarrow+\infty}\left(\Phi^{1}(\varphi)\left(t+s_{n}\right)\right)=\left(\Phi^{1} \varphi\right)(t), \quad \text { for all } t \in \mathbb{R}
$$

In a same way, we can prove that

$$
\lim _{n \rightarrow+\infty}\left(\Phi^{1}(\varphi)\left(t-s_{n}\right)\right)=(\Phi \varphi)(t), \text { for all } t \in \mathbb{R} \text {. }
$$

Thus the function $(\Phi \varphi)$ belong to $A A(\mathbb{R}, \mathbb{R})$. The proof of Lemma 3.2 is completed.

Theorem 3.1 If (H1-H5) are satisfied. Then system (3) has a unique almost automorphic solution in the region

$$
D=D\left(\varphi_{0}, \gamma\right)=\left\{\varphi \in A A\left(\mathbb{R}, \mathbb{R}^{m+n}\right),\left\|\varphi-\varphi_{0}\right\| \leq \frac{\gamma\|L\|_{\infty}}{a^{-}(1-\gamma)}\right\}
$$

where

$$
\varphi_{0}(t)=\left(\begin{array}{c}
\int_{-\infty}^{t} e^{-\int_{s}^{t} a_{11}(u) d u} L_{11}(s) d s \\
\int_{-\infty}^{t} e^{-\int_{s}^{t} a_{12}(u) d u} L_{12}(s) d s \\
\vdots \\
\int_{-\infty}^{t} e^{-\int_{s}^{t} a_{m n}(u) d u} L_{m n}(s) d s
\end{array}\right) .
$$

Proof It is easy to see that

$$
D=D\left(\varphi_{0}, \gamma\right)=\left\{\varphi \in A A\left(\mathbb{R}, \mathbb{R}^{m+n}\right), \quad\left\|\varphi-\varphi_{0}\right\| \leq \frac{\gamma\|L\|_{\infty}}{a^{-}(1-\gamma)}\right\}
$$

is a closed convex subset of $A A\left(\mathbb{R}, \mathbb{R}^{m+n}\right)$. Then

$$
\begin{aligned}
\left\|\varphi_{0}(t)\right\| & =\max _{i j \in \Lambda} \sup _{t \in \mathbb{R}}\left\|\int_{-\infty}^{t} e^{-\int_{s}^{t} a_{i j}(u) d u} L_{i j}(s) d s\right\| \\
& =\|L\|_{\infty} \max _{i j \in \Lambda} \sup _{t \in \mathbb{R}} \int_{-\infty}^{t} e^{-(t-s) a_{i j}^{-}} d s \\
& =\frac{\|L\|_{\infty}}{a^{-}} .
\end{aligned}
$$

Therefore, for any $\varphi \in D$ and by (13), we see easily that

$$
\|\varphi\| \leq\left\|\varphi-\varphi_{0}\right\|+\left\|\varphi_{0}\right\| \leq \frac{\gamma\|L\|_{\infty}}{a^{-}(1-\gamma)}+\frac{\|L\|_{\infty}}{a^{-}}=\frac{\|L\|_{\infty}}{a^{-}(1-\gamma)} .
$$

Now we prove that $\Phi$ is a self-mapping from $D$ to $D$. In fact, for arbitrary $\varphi \in D$, it follows that 


$$
\begin{aligned}
& \left\|(\Phi \varphi)(t)-\varphi_{0}(t)\right\| \\
& =\max _{i j \in \Lambda} \sup _{t \in \mathbb{R}} \| \int_{-\infty}^{t} e^{-\int_{s}^{t} a_{i j}(u) d u}\left\{\sum_{C_{k l} \in N_{r}(i, j)} C_{i j}^{k l}(s) f\left(\varphi_{k l}\left(s-\tau_{k l}(s)\right)\right) \varphi_{i j}(s)\right. \\
& \left.\quad+\sum_{C_{k l} \in N_{q}(i, j)} B_{i j}^{k l}(s) \int_{0}^{\infty} K_{i j}(u) g\left(\varphi_{k l}(s-u)\right) d u \varphi_{i j}(s)\right\} d s \| \\
& \leq \max _{i j \in \Lambda} \sup _{t \in \mathbb{R}}\left[\frac{\left(\sum_{C_{k l} \in N_{r}(i, j)}\left|C_{i j}^{k l}(t)\right| L_{f}+\frac{M}{u} \sum_{C_{k l} \in N_{q}(i, j)}\left|B_{i j}^{k l}(t)\right| L_{g}\right) \frac{\|L\|_{\infty}}{a^{-}(1-\gamma)}}{a^{-}}\right]\|\varphi\| \\
& \leq \max _{i j \in \Lambda} \sup _{t \in \mathbb{R}}\left[\frac{\left(\sum_{C_{k l} \in N_{r}(i, j)}\left|C_{i j}^{k l}(t)\right| L_{f}+\frac{M}{u} \sum_{C_{k l} \in N_{q}(i, j)}\left|B_{i j}^{k l}(t)\right| L_{g}\right)}{a^{-}}\right]\|\varphi\| \\
& \leq \frac{\gamma\|L\|_{\infty}}{a^{-}(1-\gamma)}
\end{aligned}
$$

which implies that $(\Phi \varphi) \in D$. Next, we prove the mapping $\Phi$ is a contraction mapping of $D$. In view of (H2), for any $\varphi, \psi \in D$, we have

$$
\begin{aligned}
\| & (\Phi \varphi)(t)-(\Phi \psi)(t) \| \leq \max _{i j \in \Lambda} \sup _{t \in \mathbb{R}} \int_{-\infty}^{t} e^{-\int_{s}^{t} a_{i j}(u) d u} \\
& \times\left\{\sum_{C_{k l} \in N_{r}(i, j)}\left|C_{i j}^{k l}(s)\right|\left|f\left(\varphi_{k l}\left(s-\tau_{k l}(s)\right)\right) \varphi_{i j}(s)-f\left(\psi_{k l}\left(s-\tau_{k l}(s)\right)\right) \psi_{i j}(s)\right|\right. \\
& +\sum_{C_{k l} \in N_{q}(i, j)}\left|B_{i j}^{k l}(s)\right| \mid \int_{0}^{\infty} K_{i j}(u) g\left(\varphi_{k l}(s-u)\right) d u \varphi_{i j}(s) \\
& \left.-\int_{0}^{\infty} K_{i j}(u) g\left(\psi_{k l}(s-u)\right) d u \psi_{i j}(s) \mid\right\} d s \\
\leq & \max _{i j \in \Lambda} \sup _{t \in \mathbb{R}} \int_{-\infty}^{t} e^{-\int_{s}^{t} a_{i j}(u) d u} \\
& \times\left\{\sum_{C_{k l} \in N_{r}(i, j)}\left|C_{i j}^{k l}(s)\right|\left[M_{f}\left|\varphi_{i j}(s)-\psi_{i j}(s)\right|+L_{f}\left|\varphi_{k l}\left(s-\tau_{k l}(s)\right)-\psi_{k l}\left(s-\tau_{k l}(s)\right)\right|\right]\right. \\
& +\sum_{C_{k l} \in N_{q}(i, j)}\left|B_{i j}^{k l}(s)\right|\left[\int_{0}^{\infty}\left|K_{i j}(u)\right| L_{g} d u\left|\varphi_{i j}(s)-\psi_{i j}(s)\right|\right. \\
& \left.\left.+\int_{0}^{\infty}\left|K_{i j}(u)\right| L_{g}\left|\varphi_{k l}(s-u)-\psi_{k l}(s-u)\right| \frac{\|L\| \infty}{a^{-}(1-\gamma)} d u\right]\right\} d s \\
\leq & \max _{i j \in \Lambda} \sup _{t \in \mathbb{R}} \int_{-\infty}^{t} e^{-\int_{s}^{t} a_{i j}(u) d u} \times\left\{\sum_{C_{k l} \in N_{r}(i, j)}\left|C_{i j}^{k l}(s)\right|\left(M_{f}+L_{f}\right)\right. \\
& \left.+\sum_{C_{k l} \in N_{q}(i, j)}\left|B_{i j}^{k l}(s)\right|\left(1+\frac{|| L \mid \|_{\infty}}{a^{-}(1-\gamma)}\right) L_{g} \int_{0}^{\infty}\left|K_{i j}(u)\right| d u\right\} d s\|\varphi-\psi\| \\
\leq & \max _{i j \in \Lambda} \sup _{s \in \mathbb{R}}\left\{\frac{\prod_{i j}}{a^{-}}\right\} \mid \varphi-\psi \|,
\end{aligned}
$$

where

$$
\Pi_{i j}=\sum_{C_{k l} \in N_{r}(i, j)}\left|C_{i j}^{k l}(s)\right|\left(M_{f}+L_{f}\right)+\sum_{C_{k l} \in N_{q}(i, j)}\left|B_{i j}^{k l}(s)\right|\left(1+\frac{|| L \|_{\infty}}{a^{-}(1-\gamma)}\right) L_{g} \int_{0}^{\infty}\left|K_{i j}(u)\right| d u .
$$


Then it follows from (H4) that $\Phi$ is contracting operator in $D$. Thus there exists a unique almost automorphic solution $x^{*} \in D$ of (3) that is $\Phi\left(x^{*}\right)=x^{*}$. The proof of Theorem 3.1 is completed.

\section{Exponential stability of almost automorphic solutions}

In this section, we will obtain the exponential stability of the almost automorphic solutions of system (1).

Theorem 4.1 Suppose that (H1-H5) are fulfilled. If the condition (H6)

$$
\begin{aligned}
& a_{i j_{s}}^{-}-\left\{\left[\sum_{C_{k l} \in N_{r}(i, j)} C_{i j}^{k l^{+}}\left(M_{f}+e^{\tau t} L_{f}\right)\right.\right. \\
& \left.\quad+\sum_{C_{k l} \in N_{q}(i, j)} B_{i j}^{k l+}\left[L_{g} \int_{0}^{\infty} K_{i j}(u) d u+L_{g} \frac{\|L\|_{\infty}}{a^{-}(1-\gamma)} \int_{0}^{\infty} K_{i j}(u) e^{u t} d u\right]\right\}>0
\end{aligned}
$$

holds, then the almost automorphic solution of system (3) in D is globally exponentially stable.

Proof By Theorem 3.1, we know that (3) has an almost automorphic solution $x(t)=\left(x_{11}(t), x_{12}(t), \ldots, x_{m n}(t)\right)^{T}$ with initial condition $\varphi(t)=\left(\varphi_{11}(t), \varphi_{12}(t), \ldots\right.$, $\left.\varphi_{m n}(t)\right)^{T}$. Suppose that $y(t)=\left(y_{11}(t), y_{12}(t), \ldots, y_{m n}(t)\right)^{T}$ is an arbitrary solution of (3) with initial condition $\psi(t)=\left(\psi_{11}(t), \psi_{12}(t), \ldots, \psi_{m n}(t)\right)^{T}$. Denote $u(t)=\left(u_{11}(t), u_{12}(t), \ldots, u_{m n}(t)\right)^{T}$, where $u_{i j}(t)=y_{i j}(t)-x_{i j}(t), i j \in \Lambda$. Set

$$
\begin{aligned}
\Upsilon_{i j}(t)= & t-a_{i j}+\sum_{C_{k l} \in N_{r}(i, j)} C_{i j}^{k l^{+}}\left(M_{f}+e^{\nu \tau} L_{f}\right) \\
& +\sum_{C_{k l} \in N_{q}(i, j)} B_{i j}^{k l^{+}}\left[L_{g} \int_{0}^{\infty} K_{i j}(u) d u+L_{g} \frac{\|L\|_{\infty}}{a^{-}(1-\gamma)} \int_{0}^{\infty} K_{i j}(u) e^{\nu u} d u\right] .
\end{aligned}
$$

Clearly, the functions $t \rightarrow \Upsilon_{i j}, i j \in \Lambda$, are continuous on $\mathbb{T}^{+}$and by hypothesis (H6), $\Upsilon_{i j}(0)<0$. Thus, there exists a sufficiently small constant $v$ such that $\Upsilon_{i j}(v)<0$. Take an arbitrary $\varepsilon>0$. Set

$$
z_{i j}(t)=\left|x_{i j}^{*}(t)-x_{i j}(t)\right| e^{v t}
$$

Then for all $i j \in \Lambda$, and for all $-\tau \leq t \leq 0$, one has

$$
z_{i j}(t) \leq M<M+\varepsilon
$$

Next, we shall prove that for all $t>0$,

$$
z_{i j}(t) \leq M+\varepsilon, \quad i j \in \Lambda \text {. }
$$

Suppose the contrary. Let us denote $A_{i j}=\left\{t>0, z_{i j}(t)>M+\varepsilon\right\}$. It follows that there exists $(i j)_{0} \in \Lambda$ such that $A_{(i j)_{0}} \neq \emptyset$. Let 


$$
t_{i j}=\left\{\begin{array}{l}
\inf \left(A_{i j}\right)\left\{t>0, z_{i j}(t)>M+\varepsilon\right\} \neq \emptyset \\
+\infty \quad\left\{t>0, z_{i j}(t)>M+\varepsilon\right\}=\emptyset
\end{array}\right.
$$

Clearly $t_{i j}>0$ and for all $-\tau \leq t \leq t_{i j}$. Further, one has $z_{i j}(t) \leq M+\varepsilon$. Let us denote $t_{i j_{s}}=\min _{i j \in \Lambda} t_{i j}$. It follows that $0<t_{i j_{s}}<+\infty$. and for all $-\tau \leq t \leq t_{i j_{s}}$. Note that

$$
z_{i j_{s}}\left(t_{i j_{s}}\right)=M+\varepsilon, D^{+} z_{i j_{s}}\left(t_{i j_{s}}\right) \geq 0 \text {. }
$$

Since $x_{i j}($.$) and x_{i j}^{*}($.$) are solutions of (3), we get$

$$
\begin{aligned}
& 0 \leq D^{+} z_{i j_{s}}\left(t_{i j_{s}}\right)=D^{+}\left[\left|x_{i j}^{*}(t)-x_{i j}(t)\right| e^{v t}\right]_{t=t_{i j_{s}}} \\
& =\left.e^{v t_{i j}}\left[v\left|x_{i j}^{*}(t)-x_{i j}(t)\right|+\frac{D^{+}\left|x_{i j}^{*}(t)-x_{i j}(t)\right|}{d t}\right]\right|_{t=t_{i j_{s}}} \\
& =\left|x_{i j_{s}}^{*}\left(t_{i j_{s}}\right)-x_{i j_{s}}\left(t_{i j_{s}}\right)\right| v e^{v t_{i j_{s}}}+e^{v t_{i j_{s}}} \operatorname{sgn}\left(x_{i j_{s}}^{*}\left(t_{i j_{s}}\right)-x_{i j_{s}}\left(t_{i j_{s}}\right)\right) \\
& \times\left\{-a_{i j_{s}}\left(t_{i j_{s}}\right)\left(x_{i j_{s}}^{*}\left(t_{i j_{s}}\right)-x_{i j_{s}}\left(t_{i j_{s}}\right)\right)\right. \\
& +\sum_{C_{k l} \in N_{r}(i, j)} C_{i j_{s}}^{k l}\left(t_{i j_{s}}\right)\left[f\left(x_{k l}^{*}\left(t_{i j_{s}}-\tau_{k l}\left(t_{i j_{s}}\right)\right)\right) x_{i j_{s}}^{*}\left(t_{i j_{s}}\right)\right. \\
& \left.-f\left(x_{k l}\left(t_{i j_{s}}-\tau_{k l}\left(t_{i j_{s}}\right)\right)\right) x_{i j_{s}}\left(t_{i j_{s}}\right)\right] \\
& +\sum_{C_{k l} \in N_{q}(i, j)} B_{i j_{s}}^{k l}\left(t_{i j_{s}}\right)\left[\int_{0}^{\infty} K_{i j_{s}}(u) g\left(x_{k l}^{*}\left(t_{i j_{s}}-u\right)\right) d u x_{i j_{s}}^{*}\left(t_{i j_{s}}\right)\right. \\
& \left.-\int_{0}^{\infty} K_{i j_{s}}(u) g\left(x_{k l}\left(t_{i j_{s}}-u\right)\right) d u x_{i j_{s}}\left(t_{i j_{s}}\right)\right] \\
& \leq\left|x_{i j_{s}}^{*}\left(t_{i j_{s}}\right)-x_{i j_{s}}\left(t_{i j_{s}}\right)\right| v e^{v t_{i j_{s}}}+e^{v t_{i j_{s}}}\left[-a_{i j_{s}}\left(t_{i j_{s}}\right)\left|x_{i j_{s}}^{*}\left(t_{i j_{s}}\right)-x_{i j_{s}}\left(t_{i j_{s}}\right)\right|\right. \\
& +\sum_{C_{k l} \in N_{r}(i, j)}\left|C_{i j}^{k l}\left(t_{i j_{s}}\right)\right|\left[M_{f}\left|x_{i j}^{*}\left(t_{i j_{s}}\right)-x_{i j}\left(t_{i j_{s}}\right)\right|\right. \\
& \left.+L_{f}\left|x_{k l}^{*}\left(t_{i j_{s}}-\tau_{k l}\left(t_{i j_{s}}\right)\right)-x_{k l}\left(t_{i j_{s}}-\tau_{k l}\left(t_{i j_{s}}\right)\right)\right|\right] \\
& +\sum_{C_{k l} \in N_{q}(i, j)}\left|B_{i j}^{k l}\left(t_{i j_{s}}\right)\right|\left[\int_{0}^{\infty}\left|K_{i j}(u)\right| L_{g} d u\left|x_{i j}^{*}\left(t_{i j_{s}}\right)-x_{i j}\left(t_{i j_{s}}\right)\right|\right. \\
& +\int_{0}^{\infty}\left|K_{i j}(u)\right| L_{g}\left|x_{k l}^{*}\left(t_{i j_{s}}-u\right)-x_{k l}\left(t_{i j_{s}}-u\right)\right| \frac{\|L\|_{\infty}}{a^{-}(1-\gamma)} d u \\
& \leq(M+\varepsilon)\left(v-a_{i j_{s}}\left(t_{i j_{s}}\right)\right)+\sum_{C_{k l} \in N_{r}(i, j)}\left|C_{i j}^{k l}\left(t_{i j_{s}}\right)\right| \\
& \times\left[M_{f} \mid z_{i j}\left(t_{i j_{s}}\right)+e^{\nu \tau} L_{f} z_{k l}\left(t_{i j_{s}}-\tau_{k l}\left(t_{i j_{s}}\right)\right)\right] \\
& +\sum_{C_{k l} \in N_{q}(i, j)}\left|B_{i j}^{k l}\left(t_{i j_{s}}\right)\right|\left[\int_{0}^{\infty}\left|K_{i j}(u)\right| L_{g} d u z_{i j}\left(t_{i j_{s}}\right)\right. \\
& +\int_{0}^{\infty} K_{i j}(u) e^{v u} L_{g} z_{k l}^{*}\left(t_{i j_{s}}-u\right) \frac{\|L\|_{\infty}}{a^{-}(1-\gamma)} d u \\
& \leq(M+\varepsilon)\left[\nu-a_{i j_{s}}^{-}+\sum_{C_{k l} \in N_{r}(i, j)} C_{i j}^{k l+}\left(M_{f}+e^{\nu \tau} L_{f}\right)\right. \\
& +\sum_{C_{k l} \in N_{q}(i, j)} B_{i j}^{k l}+\left[L_{g} \int_{0}^{\infty} K_{i j}(u) d u+L_{g} \frac{\|L\|_{\infty}}{a^{-}(1-\gamma)} \int_{0}^{\infty} K_{i j}(u) e^{\nu u} d u\right] .
\end{aligned}
$$


It follows that

$$
\begin{aligned}
& v-a_{i j_{s}}^{-}+\sum_{C_{k l} \in N_{r}(i, j)} C_{i j}^{k l+}\left(M_{f}+e^{v \tau} L_{f}\right) \\
& +\sum_{C_{k l} \in N_{q}(i, j)} B_{i j}^{k l}+\left[L_{g} \int_{0}^{\infty} K_{i j}(u) d u+L_{g} \frac{\|L\|_{\infty}}{a^{-}(1-\gamma)} \int_{0}^{\infty} K_{i j}(u) e^{v u} d u \geq 0 .\right.
\end{aligned}
$$

Then $\Upsilon_{i j}(v) \geq 0$ which contradicts the fact that $\Upsilon_{i j}(v)<0$. Thus we obtain that

$$
z_{i j}(t)=\left|x_{i j}(t)-\varphi_{i j}(t)\right| \leq(M+\varepsilon) e^{-v t}, \quad \text { for all } t>0 .
$$

Note that $\left\|x(t)-x_{i j}^{*}(t)\right\|=\max _{i j \in \Lambda}\left|x_{i j}(t) x_{i j}^{*}(t)\right|$, then letting $\varepsilon \rightarrow 0$, we obtain

$$
\left|x(t)-x_{i j}^{*}(t)\right| \leq M e^{-v t}, \quad \text { for all } t>0 .
$$

which means that the almost automorphic solution of (3) is globally exponentially stable. The proof of Theorem 4.2 is completed.

Remark 4.1 Shao (2008) studied the anti-periodic solutions of system (1) with the $B_{i j}(t)=0, a_{i j}(t)=a_{i j}$ and $\tau_{k l}=\tau(t)$. Peng and Huang (2009) investigated the existence and exponential stability of anti-periodic solutions for model (1) with $C_{i j}(t)=0$ and $a_{i j}(t)=a_{i j}$. Zhao et al. (2010) considered anti-periodic solutions of model (1) with the $B_{i j}(t)=0$ and $\tau_{k l}=\tau(t)$. Peng and Wang (2011) analyzed the anti-periodic solutions for (1) with time-varying delays $\sigma_{i j}(t)$ in leakage terms. Zhou et al. (2006a) discussed the existence and stability of almost periodic solutions for model (1) with $C_{i j}(t)=0$. Li and Wang (2012) focused on the almost periodic solutions for model (1) with $C_{i j}(t)=0$ on time scales. In addition, there are many papers that have investigated almost periodic solutions or convergence behavior of the special form or a more general form of model (1). We refer the reader to (Zhao and Zhang 2008; Cai et al. 2008; Huang and Cao 2003; Ding et al. 2008; Liu and Huang 2006, 2007; Liu 2007, 2009a, b; Fan and Shao 2010; Liu et al. 2006; Shao et al. 2009; Xia et al. 2007; Zhou et al. 2006b; Liu and Ding 2014; Li and Wang 2012; Li et al. 2008; Meng and Li 2008; Li and Huang 2008). In this paper, we consider the almost automorphic solutions of (1), which complement with some previous studies in (Shao 2008; Peng and Huang 2009; Zhao et al. 2010; Peng and Wang 2013; Zhou et al. 2006a; Zhao and Zhang 2008; Cai et al. 2008; Huang and Cao 2003; Ding et al. 2008; Liu and Huang 2007; Liu 2007, 2009a, b; Fan and Shao 2010; Liu and Huang 2006; Liu et al. 2006; Shao et al. 2009; Xia et al. 2007; Zhou et al. 2006b; Liu and Ding 2014; Li and Wang 2012; Li et al. 2008; Meng and Li 2008; Li and Huang 2008).

Remark 4.2 In Li and Yang (2014), authors considered the almost automorphic solutions for neutral type neural networks with delays in leakage on time ccales, in Abbas et al. (2014), authors considered the almost automorphic solutions for neural networks with impulses. All the methods can not be applied to this paper to obtained our results in this paper. Therefore our results are completely new. 


\section{Numerical example}

In this section, we will give an example to illustrate the feasibility and effectiveness of our main results obtained in previous sections. Considering the following shunting inhibitory cellular neural networks with time-varying delays

$$
\left\{\begin{aligned}
x_{11}^{\prime}(t)= & -a_{11}(t) x_{11}(t)+\sum_{C_{k l} \in N_{r}(1,1)} C_{11}^{k l}(t) f\left(x_{k l}\left(t-\tau_{k l}(t)\right)\right) x_{11}(t) \\
& +\sum_{C_{k l} \in N_{q}(1,1)} B_{11}^{k l}(t) \int_{0}^{\infty} K_{11}(u) g\left(x_{k l}(t-u)\right) d u x_{11}(t)+L_{11}(t), \\
x_{12}^{\prime}(t)= & -a_{12}(t) x_{12}(t)+\sum_{C_{k l} \in N_{r}(1,2)} C_{12}^{k l}(t) f\left(x_{k l}\left(t-\tau_{k l}(t)\right)\right) x_{12}(t) \\
& +\sum_{C_{k l} \in N_{q}(1,2)} B_{12}^{k l}(t) \int_{0}^{\infty} K_{12}(u) g\left(x_{k l}(t-u)\right) d u x_{12}(t)+L_{12}(t), \\
x_{21}^{\prime}(t)= & -a_{21}(t) x_{21}(t)+\sum_{C_{k l} \in N_{r}(2,1)} C_{21}^{k l}(t) f\left(x_{k l}\left(t-\tau_{k l}(t)\right)\right) x_{21}(t) \\
& +\sum_{C_{k l} \in N_{q}(2,1)} B_{21}^{k l}(t) \int_{0}^{\infty} K_{21}(u) g\left(x_{k l}(t-u)\right) d u x_{21}(t)+L_{21}(t), \\
x_{22}^{\prime}(t)= & -a_{22}(t) x_{22}(t)+\sum_{C_{k l} \in N_{r}(2,2)} C_{22}^{k l}(t) f\left(x_{k l}\left(t-\tau_{k l}(t)\right)\right) x_{22}(t) \\
& +\sum_{C_{k l} \in N_{q}(2,2)} B_{22}^{k l}(t) \int_{0}^{\infty} K_{22}(u) g\left(x_{k l}(t-u)\right) d u x_{22}(t)+L_{22}(t),
\end{aligned}\right.
$$

where $f(u)=0.5(|u+1|-|u-1|), K_{i j}=\cos \left(\frac{1}{2+\sin t+\sin \sqrt{2} t}\right)$ and

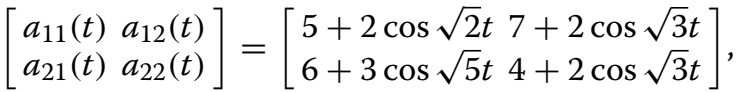

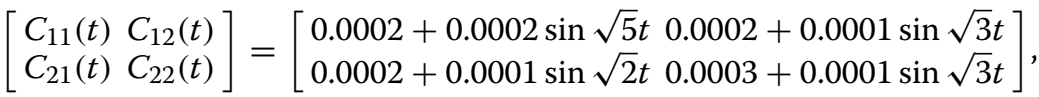

$$
\begin{aligned}
& {\left[\begin{array}{ll}
B_{11}(t) & B_{12}(t) \\
B_{21}(t) & B_{22}(t)
\end{array}\right]=\left[\begin{array}{ll}
0.0003+0.0001 \sin \sqrt{2} t & 0.0003+0.0001 \sin \sqrt{3} t \\
0.0002+0.0001 \sin \sqrt{5} t & 0.0002+0.0001 \sin \sqrt{5} t
\end{array}\right],} \\
& {\left[\begin{array}{ll}
L_{11}(t) & L_{12}(t) \\
L_{21}(t) & L_{22}(t)
\end{array}\right]=\left[\begin{array}{ll}
0.002+0.002 \cos \sqrt{3} t & 0.003+0.002 \cos \sqrt{7} t \\
0.002+0.002 \cos \sqrt{7} t & 0.001+0.002 \cos \sqrt{3} t
\end{array}\right] .}
\end{aligned}
$$

Let $\quad r=q=1, \tau_{k l}(t)=0.005$. Then $\quad$ we get $\quad L_{f}=L_{g}=M_{g}=M_{f}=1, a^{-}=2$, $\|L\|_{\infty}=0.005, K_{i j}(t) \leq e^{-t}, M=u=1, \tau=0.005$ and

$$
\begin{aligned}
& {\left[\begin{array}{llll}
\sum_{C_{k l} \in N_{1}(1,1)} & C_{11}^{k l}+ & \sum_{C_{k l} \in N_{1}(1,2)} C_{12}^{k l+} \\
\sum_{C_{k l} \in N_{1}(2,1)} & C_{21}^{k l} & \sum_{C_{k l} \in N_{1}(2,2)} & C_{22}^{k l}
\end{array}\right]=\left[\begin{array}{ll}
0.0014 & 0.0014 \\
0.0014 & 0.0014
\end{array}\right],} \\
& {\left[\begin{array}{ll}
\sum_{C_{k l} \in N_{1}(1,1)} B_{11}^{k l}+ & \sum_{C_{k l} \in N_{1}(1,2)} B_{12}^{k l}{ }^{+} \\
\sum_{C_{k l} \in N_{1}(2,1)} B_{21}^{k l}+ & \sum_{C_{k l} \in N_{1}(2,2)} B_{22}^{k l}
\end{array}\right]=\left[\begin{array}{ll}
0.0016 & 0.0016 \\
0.0016 & 0.0016
\end{array}\right] .}
\end{aligned}
$$


Hence

$$
\begin{aligned}
& \gamma= \max _{i j \in \Lambda} \sup _{t \in \mathbb{R}}\left\{\frac{\sum_{C_{k l} \in N_{1}(i, j)}\left|C_{i j}^{k l}(t)\right| L_{f}+\frac{M}{u} \sum_{C_{k l} \in N_{1}(i, j)}\left|B_{i j}^{k l}(t)\right| L_{g}}{a^{-}}\right\} \\
& \leq \frac{0.0014+0.0016}{2}=0.0015<1, \\
& \frac{\|L\|_{\infty}}{a^{-}(1-\gamma)}= \frac{0.005}{1(1-0.0015)}=\frac{10}{17}<1, \\
& \Pi_{i j}= \sum_{C_{k l} \in N_{1}(i, j)}\left|C_{i j}^{k l}(s)\right|\left(M_{f}+L_{f}\right) \\
&+\sum_{C_{k l} \in N_{1}(i, j)}\left|B_{i j}^{k l}(s)\right|\left(1+\frac{\|L\|_{\infty}}{a^{-}(1-\gamma)}\right) L_{g} \int_{0}^{\infty}\left|K_{i j}(u)\right| d u \\
& \leq 0.0014 \times 2+0.0016 \times 0.6=0.00376, \\
& \max _{i j \in \Lambda} \sup _{s \in \mathbb{R}}\left\{\frac{\Pi_{i j}}{\left.a^{-}\right\}=}\right. 0.00188<1, \\
& a_{i j_{s}}^{-}-\left[\sum_{C_{k l} \in N_{1}(i, j)} C_{i j}^{k l+}\left(M_{f}+e^{\tau t} L_{f}\right)-\sum_{C_{k l} \in N_{1}(i, j)} B_{i j}^{k l}+\left[L_{g} \int_{0}^{\infty} K_{i j}(u) d u\right.\right. \\
&\left.+L_{g} \frac{\|L\|_{\infty}}{a^{-}(1-\gamma)} \int_{0}^{\infty} K_{i j}(u) e^{u t} d u\right]=1.000624>0 .
\end{aligned}
$$

Thus all assumptions in Theorems 4.1 and 4.2 are fulfilled. Thus we can conclude that (27) has an almost automorphic solution, which is globally exponentially stable. The results are verified by the numerical simulations in Fig. 1.

\section{Conclusions}

In this paper, we consider a class of shunting inhibitory cellular neural networks with time-varying delays. Some sufficient conditions for the existence and exponential stability of almost automorphic solutions for the shunting inhibitory cellular neural networks

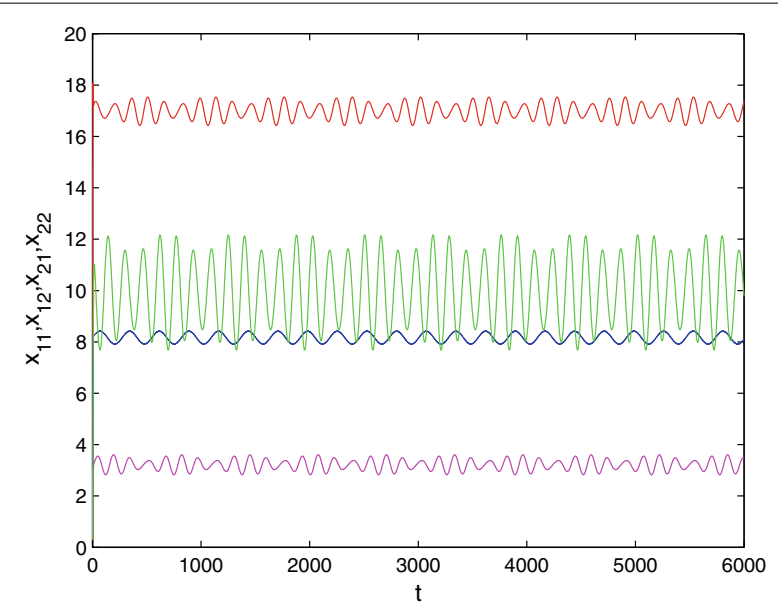

Fig. 1 Time response of state variables $x_{i j}(i, j=1,2)$, where the red line stands for $x_{11}$, the magenta line stands for $x_{12}$ ", the blue line stands for $x_{21}$ and the green line stands for $x_{22}$ 
with time-varying delays have been established. It is shown that the time delay has no effect on the existence of almost automorphic solutions for system (1) but has important effect on the global exponential stability of almost automorphic solutions for system (1). To the best of our knowledge, it is the first time to deal with the almost automorphic solution for the shunting inhibitory cellular neural networks with time-varying delays. Moreover, our criteria are easy to check and apply in practice and are of prime importance and great interest in many application fields and the designs of networks. Our results complement with some previous ones. The method of this paper can be applied directly to many other neural networks, such as BAM neural networks, Hopfield neural networks and so on.

Authors' contributions

Both authors have made the same contribution. Both authors read and approved the final manuscript.

\section{Author details}

${ }^{1}$ Guizhou Key Laboratory of Economics System Simulation, Guizhou University of Finance and Economics, Guiyang 550004, People's Republic of China. ${ }^{2}$ School of Mathematics and Physics, University of South China, Hengyang 421001, People's Republic of China.

\section{Acknowledgements}

The first author was supported by National Natural Science Foundation of China (No.11261010), Natural Science and Technology Foundation of Guizhou Province(J[2015]2025) and 125 Special Major Science and Technology of Department of Education of Guizhou Province ([2012]011). The second author was supported by National Natural Science Foundation of China (No.11101126). The authors would like to thank the referees and the editor for helpful suggestions incorporated into this paper.

\section{Competing interests}

The authors declare that they have no competing interests.

Received: 20 September 2015 Accepted: 3 November 2015

Published online: 24 November 2015

\section{References}

Abbas A, Mahto L, Hafayed M, Alimi AM (2014) Asymptotic almost automorphic solutions of impulive neural network with almost automorphic coefficients. Neurocomputing 142:326-334

Bochner S (1962) A new approach to almost periodicity. Proc Natl Acad Sci USA 48(12):195-205

Bouzerdoum A, Pinter RB (1993) Shunting inhibitory cellular neural networks: derivation and stability analysis. IEEE Trans Circuits Syst I Fund Theory Appl 40(3):215-221

Cai MS, Zhang H, Yuan ZH (2008) Positive almost periodic solutions for shunting inhibitory cellular neural networks with time-varying delays. Math Comput Simul 78(4):548-558

Chen L, Zhao HY (2008) Global stability of almost periodic solution of shunting inhibitory cellular neural networks with variable coefficients. Chaos Solitons Fractals 35(2):351-357

Chérif F (2014) Sufficient conditions for global stability and existence of almost automorphic solution of a class of RNNs. Diff Equat Dyn Sys 22(2):191-207

Chérif F, Nahia ZB (2013) Global attractivity and existence of weighted pseudo almost automorphic solution for GHNNs with delays and variable coefficients. Gulf J Math 1(1):5-24

Chua LO, Roska T(1990) Cellular neural networks with nonlinear and delay-type template elements. In: Proceedings of the 1990 IEEE International Workshop on Cellular Neural Networks and Their Applications, pp 12-25

Chua LO, Yang L (1988a) Cellular neural networks: theory. IEEE Trans Circuits Syst 35(10):1257-1272

Chua LO, Yang L (1988b) Cellular neural networks: application. IEEE Trans Circuits Syst 35(10):1273-1290

Cuevas C, Henríquez HR, Lizama C (2012) On the existence of almost automorphic solutions of Volterra difference equations. J Differ Equ Appl 18(11):1931-1946

Diagana T, Henriquez HR, Hernández EM (2008) Almost automorphic mild solutions to some partial neutral functionaldifferential equations and applications. Nonlinear Anal TMA 69(5-6):1485-1493

Ding HS, Liang J, Xiao TJ (2008) Existence of almost periodic solutions for shunting inhibitory cellular neural networks with time-varying delays. Phys Lett A 372(33):5411-5416

Ezzinbi K, Fatajou S, N'Guérékata GM (2007) Almost automorphic solutions for some partitial functional differential equations. J Math Anal Appl 328(1):344-358

Fan QY, Shao JY (2010) Positive almost periodic solutions for shunting inhibitory cellular neural networks with time-varying and continuously distributed delays. Commun Nonlinear Sci Numer Simul 15(6):1655-1663

Goldstein JA, N'Guérékata GM (2005) Almost automorphic solutions of semilinear evolution equations. Proc Amer Math Soc 133(8):2401-2408

Hale JK (1977) Theory of functions differential equations. Springer, New York 
Hilger S (1990) Analysis on measure chains-a unified approach to continuous and discrete calculus. Results Math $18(1-2): 18-56$

Huang ZD, Peng LQ, Xu M (2010) Anti-periodic solutions for high-order cellular neural netowrks with time-varying delays. Electron J Diff Eq. 5:1-9

Huang X, Cao JD (2003) Almost periodic solution of shunting inhibitory cellular neural networks with time-varying delays. Phys Lett A 314(3):222-231

Li YQ, Huang LH (2008) Exponential convergence behavior of solutions to shunting inhibitory cellular neural networks with time-varying coefficients. Math Comput Model 48(3-4):499-504

LiYQ, Meng H, Zhou QY (2008) Exponential convergence behavior of shunting inhibitory cellular neural networks with time-varying coefficients. J Comput Appl Math 216(10):164-169

Liu YG, You ZS, Cao LP (2006) On the almost periodic solution of generalized shunting inhibitory cellular neural networks with continulusly distributed delays. Phys Lett A 360(1):122-130

Liu BW (2007) Almost periodic solutions for shunting inhibitory cellular neural networks without global Lipschitz activation functions. J Comput Appl Math 203(1):159-168

Liu BW (2009) Stability of shunting inhibitory cellular neural networks with unbounded time-varying delays. Appl Math Lett 22(1):1-5

Liu BW (2009) New convergence behavior of solutions to shunting inhibitory cellular neural networks with unbounded delays and time-varying coefficients. Appl Math Model 33(1):54-60

Liu QL, Ding HS (2014) Existence and stability of almost periodic solutions for SICNNs with neuarl type delays. Electron J Diff Eq. 23:1-14

Liu BW, Huang LH (2006) Existence and stability of slmost periodic solutions for shunting inhibitory cellular neural networks with continulusly distributed delays. Phys Lett A 349(3-4):177-186

Liu BW, Huang LH (2007) Almost periodic solutions for shunting inhibitory cellular neural networks with time-varying delays. Appl Math Lett 20(1):70-74

Li YK, Wang C (2012) Almost periodic solutions of shunting inhibitory cellular neural networks on time scales. Commun Nonlinear Sci Numer Simul 17(8):3258-3266

Li YK, Yang L (2014) Almost automorphic solution for nautral type high-order Hopfied neural networks with delays in leakage terms on time scales. Appl Math Comput 242:679-693

Lizama C, Mesquita JG (2013) Almost automorphic solutions of dynamic equations on time scales. J Funct Anal 265(10):2267-2311

Meng H, Li YQ (2008) New convergence behavior of shunting inhibitory cellular neural networks with time-varying coefficients. Appl Math Lett 21(7):717-721

N'Guérékata GM (2004) Existence and uniqueness of almost automorphic mild solutions to some semilinear abstract differential equations. Semigroup Forum 69(1):80-86

N'Guérékata GM (2005) Topics in Almost Automorphy. Springer, New York

Peng GQ, Huang LH (2009) Anti-periodic solutions for shunting inhibitory cellular neural networks with continuously distributed delays. Nonlinear Anal Real World Appl 10(4):2434-2440

Peng LQ, Wang WT (2013) Anti-periodic solutions for shunting inhibitory cellular neural networks with time-varying delays in leakage terms. Neurocomputing 111:27-33

Roska T, Chua LO (1992) Cellular neural networks with nonlinear and delay-type templates. Int J Circuit Theory Appl 20(5):469-481

Shao JY (2008) Anti-periodic solutions for shunting inhibitory cellular neural networks with time-varying delays. Phys Lett A 372(30):5011-5016

Shao JY, Wang LJ, Ou CX (2009) Almost periodic solutions for shunting inhibitory cellular neural networks without global Lipschitz activation functions. Appl Math Model 33(6):2575-2581

Song XL, Xin XL, Huang WP (2012) Exponential stability of delayed and impulsive cellular neural networks with partially Lipschitz continuous activation functions. Neural Netw 29-30:80-90

Wang LX, Zhang JM, Shao HJ (2014) Existence and global stability of a periodic solution for a cellular neural network. Commun Nonlinear Sci Numer Simul 19(9):2983-2992

Wang JL, Jiang HJ, Hu C, Ma TL (2014) Convergence behavior of delayed discrete cellular neural network without periodic coefficients. Neural Netw 53:61-68

Wang C, Li YK (2013) Weighted pseudo almost automorphic functions with applications to abstract dynamic equations on time scales. Ann Polon Math 108(3):225-240

Xia YH, Cao JD, Huang ZK (2007) Existence and exponential stability of almost periodic solution for shunting inhibitory cellular neural networks with impulses. Chaos Solitons Fractals 34(5):1599-1607

Yang YQ, Cao JD (2007) Stability and periodicity in delayed cellular neural networks with impulsive effects. Nonlinear Anal Real World Appl 8(1):362-374

Zhang AP (2013) Existence and exponential stability of anti-periodic solutions for HCNNs with time-varying leakage delays. Adv Diff Eq. 162:1-16

Zhang H, Shao JY (2013) Existence and exponentially stability of almost periodic solutions for CNNs with time-varying leakage delays. Neurocomputing 121:226-233

Zhao CH, Fan QY, Wang WT (2010) Anti-periodic solutions for shunting inhibitory cellular neural networks with timevarying coefficients. Neural Process Lett 31(3):259-267

Zhao W, Zhang HS (2008) On almost solution of shunting inhibitory cellular neural networks with variable coefficients and time-varying delays. Nonlinear Anal Real World Appl 9(5):2326-2336

Zhou QY, Xiao B, Yu YH (2006) Existence and stability of almost periodic solutions for shunting inhibitory cellular neural networks with continuously distributed delays. Electron J Diff Eq. 9:1-10

Zhou TJ, Liu YR, Chen AP (2006) Almost periodic solution for shunting inhibitory cellular neural networks with timevarying delyas and variable coefficents. Neural Process Lett 23(3):243-255 